\title{
Documentation of Procedural Sedation by Emergency Physicians
}

This article was published in the following Dove Press journal:

Drug, Healthcare and Patient Safety

\author{
Mischa Veen (iD) \\ Peer van der Zwaal ${ }^{2}$ \\ M Christien van der Linden \\ 'Department of Emergency Medicine, \\ Haaglanden Medical Center, The Hague, \\ the Netherlands; ${ }^{2}$ Department of \\ Orthopaedic Surgery, Haaglanden \\ Medical Center, The Hague, the \\ Netherlands
}

\begin{abstract}
Introduction: Patients presenting to the emergency department (ED) frequently require procedural sedation and analgesia (PSA) to facilitate procedures, such as joint reduction. Proper documentation of screening demonstrates awareness of the necessity of presedation assessment. It is unknown if introducing emergency physicians (EPs) at the ED improves presedation assessment and documentation. In this study the differences in documentation of ED sedation and success rates for reduction of hip dislocations in the presence versus absence of EPs are described.
\end{abstract}

Methods: In this retrospective descriptive study, we analyzed data of patients presenting with a dislocated hip post total hip arthroplasty (THA) shortly after the introduction of EPs. The primary outcome measure was the presence of documentation of presedation assessment. Secondary outcomes were documentation of medication, vital signs, and success rate of hip reductions.

Results: In the two-year study period, 133 sedations for hip reductions were performed. Sixty-eight sedations were completed by an EP. The documentation of fasting status, airway screening, analgesia use, and vital signs was documented significantly more often when an EP was present (respectively $64.9 \%, 80.3 \%, 37.4 \%$, and $72.7 \%$, all $P<0.001$ ). There was no difference in success rate of hip reductions between the groups.

Conclusion: PSA in the ED is associated with superior documentation of presedation assessment, medication, and vital signs when EPs are involved.

Keywords: conscious sedation, emergency department, sedation, analgesia

\section{Introduction}

Procedural sedation and analgesia (PSA) benefits painful procedures in the emergency department (ED) which are not expected to be successful without pharmacological support. In these situations PSA is a means to avoid unnecessary admission and anesthesia. ${ }^{1-4}$

PSA is an integral part of emergency medicine (EM) practice. ${ }^{5,6}$ Studies demonstrate the safety of PSA when performed by trained emergency physicians (EPs). ${ }^{1,3,4,7,8}$

There are few studies about the quality of documentation of PSA in the ED. Documentation of ED procedures, such as sedation, is generally described as inadequate. Documentation of PSA in the ED contains an unsatisfactory amount of information on airway screening and the occurrence of adverse events. ${ }^{9,10}$ Although inadequate documentation does not mean that the procedure was done poorly, ensuring good documentation may improve patient safety. ${ }^{9,10}$ Previous studies demonstrated slight improvements of documentation after introducing an
Correspondence: Mischa Veen Department of Emergency Medicine, Haaglanden Medical Center, Post Office Box 432, The Hague, 250 I CK, the Netherlands

Tel +31 - $88-9797900$ extension 6868

$\mathrm{Fax}+31-703125976$

Email m.veen@haaglandenmc.nl

Drug, Healthcare and Patient Safety 2021:13 95-100 
educational program and a preprinted form to assist documentation. ${ }^{9,10}$ However, the improvements were often minor and inconsistent. One of the suggested reasons for the poor improvement was that part of the staff had not partaken in the educational program. ${ }^{9,10}$

It is unknown how the introduction of EPs affects the adequacy of documentation of PSA.

In the Netherlands there are still too few EPs to staff all EDs around the clock. ${ }^{5}$ There are eighty-seven Dutch EDs providing twenty-four hour care. Only twenty EDs have a day-and-night presence of EPs. In twelve EDs there are no EPs present at all. ${ }^{6,11}$

After a merger of our two hospitals, EPs of one hospital were newly introduced to the ED of the other hospital. The latter ED employed junior doctors who commonly worked with distant supervision. This generally involved supervision by telephone from a non-EM specialist. ${ }^{5,12,13}$ Initially the EPs only covered office hours at their "new" location. Gradually the EPs started introducing a different culture. ${ }^{5}$ One of the most prominent changes the EPs introduced was the structured approach to PSA including screening of the airway and enquiring about the fasting status, using a standard and structured PSA form. ${ }^{5,6,14,15}$

Given the differences in the approach to PSA the ED crew noticed, we conducted a study to objectify these variations. In this study we describe the differences in documentation of PSA in the presence versus the absence of EPs. With the introduction of EPs we expected an overall improvement in the quality of documentation.

\section{Materials and Methods}

\section{Setting}

This study is a single-center retrospective descriptive analysis of sedations performed on patients presenting to the ED between January 2015 and December 2016 with hip dislocations and a history of total hip arthroplasty (THA). The start of this period coincided with the introduction of EPs at the study site. The study was performed in the ED of an urban city hospital, with an annual patient census of 20,000 visits. This ED focused on elderly patients and those with suspected neck of femur fractures.

\section{Sampling}

We included sedations for the dislocation of artificial hips, which is among the most frequent indications for PSA., ${ }^{5,12}$ Hip dislocations are readily identifiable in the electronic patient system. In addition, hip dislocations usually require sedation as part of their treatment. Other presenting problems, such as shoulder dislocations, require PSA less frequently. Dislocated hips usually involve elderly patients in whom careful presedation assessment is prudent.

\section{Measurements and Outcomes}

The primary outcomes of this study were the differences in documentation of presedation assessment (fasting status and screening for a potentially difficult airway) between the situation in which an EP was present and in the absence of EPs (the no-EP group).

Our secondary outcomes were the variations in type of medication, the documentation of procedural vital signs, and the success rate of hip reductions.

\section{Data Collection}

All adult patients requiring sedation for a hip dislocation were eligible. Patients were identified using the electronic patient system. The patients' presenting complaints were screened for terms such as "dislocated hip", "hip fracture", and "painful hip". Those patients for whom no sedation was used to reduce a dislocation in the ED were excluded from the analysis. When a patient had been sedated, wokeup and consequently received a second sedation, this was considered as two separate sedations both included separately. An extra bolus of sedative to prolong an ongoing sedation was not considered a new sedation.

\section{Data Analysis}

Continuous outcomes were reported as medians with interquartile range (IQR). Categorical data were analyzed using Chi-squared tests. Age was analyzed with the MannWhitney $U$-test, and the Fisher's exact test was used for the documentation of medication. A $P$-value of $<0.05$ was considered statistically significant. Statistical analysis were performed on IBM SPSS Statistics version 22 (Armonk, New York, USA).

\section{Ethics}

The Ethical Review Board decided that the study did not fall under the scope of the Medical Research Involving Human Subjects Act (WMO) because of its retrospective nature (METC, Southwest Holland, nr. 17-026).

\section{Results}

In 2015 and 2016 there were 38.785 ED presentations. During this period 154 patients with a dislocated hip were identified, resulting in 133 sedations (Figure 1). An 


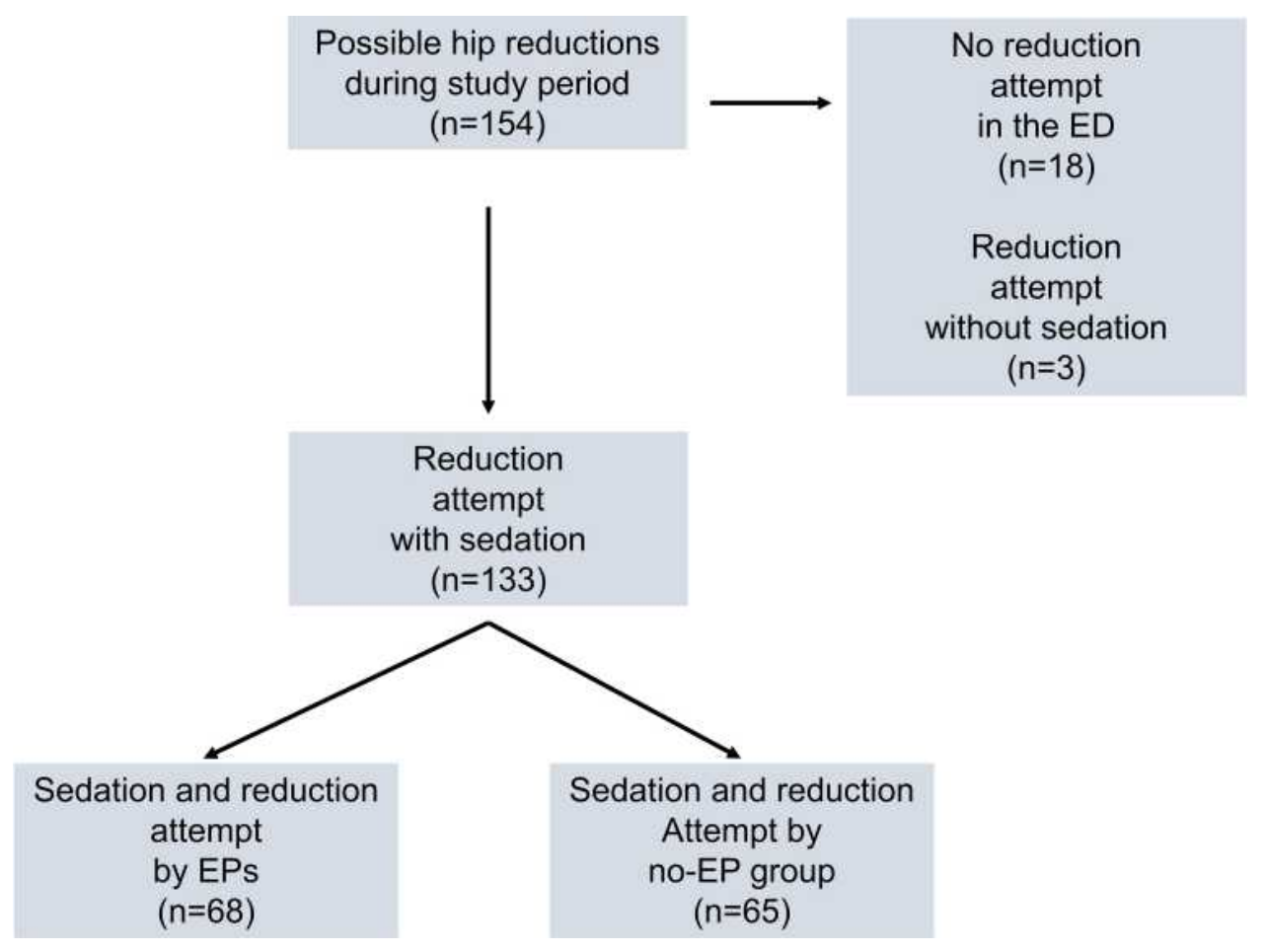

Figure I Flow chart of included sedations.

Abbreviations: EP, emergency physician; no-EP, no emergency physician present.

EP was involved in 68 of these sedations. The remaining 65 sedations were performed in the absence of EPs.

Table 1 describes characteristics of the sedation cases that were completed with an EP present and those without. The median age of the patients per sedation was 77.5 years, $74.4 \%$ of sedations involved patients over the age of seventy, and $75.9 \%$ was female. These results were not significantly different between the EP and no-EP group. Sixty-six out of 133 sedations (49.6\%) were performed during off-hours and mostly done by the no-EP group. Forty-nine of the 133 sedation concerned patients who

Table I Demographic and Clinical Characteristics of Sedation Cases $(n=133)$

\begin{tabular}{|l|l|l|}
\hline Characteristics & $\begin{array}{l}\text { EP Present } \\
(\mathbf{n}=68)\end{array}$ & $\begin{array}{l}\text { No-EP Present } \\
(\mathbf{n}=65)\end{array}$ \\
\hline Median age in years [IQR] & $77.5[70.6-87.3]$ & $77.5[64.9-85.5]$ \\
Age $>70$ years (\%) & $55(80.9)$ & $44(67.7)$ \\
Female (\%) & $50(73.5)$ & $51(78.5)$ \\
Sedation in off-hours (\%) & $13(19.1)$ & $53(81.5)^{*}$ \\
ASA Physical Status & $18(26.5)$ & $31(47.7)^{* *}$ \\
Classification $\geq$ III (\%) & & \\
\hline
\end{tabular}

Notes: *Statistically significant difference, $* P<0.001$; **Statistically significant difference, $P=0.01$.

Abbreviations: IQR, interquartile range; ASA, American Society for Anesthesiologists; EP, emergency physician. were ASA class $\geq$ III. Significantly more of these patients were sedated in the absence of EPs $(P=0.01)$.

Study outcomes on documentation items are presented in Table 2. A standard PSA form was used $78.8 \%$ more often in the presence of an EP $(P<0.001)$. The fasting status was documented $64.9 \%$ more frequently by EPs $(P<0.001)$. Screening for potential airway difficulties was reflected in the notes $80.3 \%$ more often when EPs were involved $(P<0.001)$.

The type of analgesia used was recorded $37.4 \%$ less frequently when EPs were not involved in the sedation $(P<0.001)$. Further analysis of analgesia use revealed that EPs had a clear preference for fentanyl, which they used in

Table 2 Documentation-Items of Sedation Cases $(n=133)$

\begin{tabular}{|l|l|l|}
\hline Documentation Items & $\begin{array}{l}\text { EP Present } \\
\mathbf{n = 6 8}\end{array}$ & $\begin{array}{l}\text { No-EP Present } \\
\mathbf{n}=65\end{array}$ \\
\hline $\begin{array}{l}\text { PSA form used (\%) } \\
\text { Fasting status documented (\%) }\end{array}$ & $63(92.6)$ & $9(13.8)^{*}$ \\
Airway screening & $63(92.6)$ & $18(27.7)^{*}$ \\
documented (\%) & $8(12.3)^{*}$ \\
Analgesia documented (\%) & $61(89.7)$ & $34(52.3)^{*}$ \\
Sedative documented (\%) & $68(100)$ & $62(95.4)$ \\
Vital signs documented (\%) & $62(91.2)$ & $12(18.5)^{*}$ \\
\hline
\end{tabular}

Note: *Statistically significant difference, $P<0.001$

Abbreviations: PSA, procedural sedation and analgesia; EP, emergency physician. 
$80.9 \%(55 / 68)$ of their sedations. Piritramide was used in $4.4 \%$ (3/68), and there was incidental use of other medications or a combination of those. In the no-EP group piritramide was used in $24.6 \%$ (16/65) and fentanyl in $16.9 \%$ $(11 / 65)$ of sedations. There was use of morphine, esketamine, alfentanil, paracetamol, or a combination of these analgesics in $10.8 \%(7 / 65)$ of no-EP sedations.

There was no significant difference between the EP and the no-EP groups in the documentation of the sedative. EPs used propofol in $89.7 \%(61 / 68)$, midazolam in $8.8 \%(6 / 68)$, and diazepam in $1.5 \%(1 / 68)$ of their sedations. The no-EP group used midazolam in 61.5\% (40/65), diazepam in $26.2 \%$ (17/65), propofol in 6.2\% (4/65), and a diazepam-midazolam combination in $1.5 \%(1 / 65)$. Vital signs were recorded $72.7 \%$ less often in the no-EP group $(P<0.001)$.

The overall reduction outcome did not differ significantly and was successful in 80.9\% (55/68) when an EP was present, and was $80.0 \%$ successful $(52 / 65)$ when EPs were not present.

\section{Discussion}

To our knowledge, this is the first study in which the documentation of ED sedation for hip dislocations post THA was assessed. Little is known about the adequacy of documentation for PSA. ${ }^{9}$ Our study suggests that EPs are superior in documenting PSA in the ED. Although adequate documentation does not guarantee that the procedure is done perfect, proper documentation of sedation suggests that the provider has knowledge of PSA.

Some EDs are not staffed with EPs and consequently may not have adopted a structured and standard approach to PSA. EDs without EPs are often staffed with junior doctors supervised by non-EM consultants, which are generally not physically present in the off-hours. The junior doctors, providing direct care, may lack training and experience in sedation. ${ }^{5,13}$ This is a realistic concern for patients with hip dislocations, an elderly population with high ASA classification.

Without proper teaching and without direct supervision patients are potentially exposed to inferior screening, insufficient analgesia, or inadequate sedation. ${ }^{14,16}$ Clinicians providing PSA should be familiar with the sedation-oriented aspects of the patient's medical history, their anatomy, and how these might alter the patient's response to sedation, as mandated by current guidelines. ${ }^{17}$

Screening for possible risk factors should be considered standard preparation for PSA..$^{2,15,17-21}$ In our study, the documentation of such items is clearly superior in the presence of EPs, showing that the practitioner is aware of their importance.
The same is true for the registration of analgesia and documentation of vital signs during the procedure. Both are important parts of registration and should be strongly encouraged. ${ }^{1,2}$

In the last two decades Dutch EPs started using sedatives such as propofol, (es)ketamine and etomidate for PSA as opposed to benzodiazepines which have a longer standing tradition in the ED. Initially, there was considerable concern and resistance to the use of ultra-short working agents carried out by EPs. ${ }^{14,18}$ However, there is superfluous literature supporting the claim that this practice is safe. ${ }^{1,7,8,18,19}$ Our results confirm that EPs have a preference for ultra-short working agents. Nevertheless, benzodiazepines are still commonly used in their absence.

Proper documentation and protocols are expected to lead to better outcomes for patients. Although there was no significant difference in success rate in our study, there are still important reasons for having good documentation such as the medicolegal implications in case of adverse events.

Our single-site, retrospective, descriptive study has limitations such as the small number of non-randomized patients included. Our study specifically included procedures and not patients. Patients could be included several times and included in both the EP and the no-EP group on different occasions. This should be considered when interpreting the characteristics of the population. Inclusion of cases was performed by manually screening the electronic patient system for patients with possible hip dislocations. This method leaves the possibility of missed inclusions. There was one data abstractor who was not blinded. The junior doctors that make up the no-EP group performed most of their sedations in the off-hours. Perhaps this also influenced the documentation of their sedations. We feel that the use of a standard PSA form, such as the one introduced by the EPs, improves the quality of documentation in case of sedation, although this is not necessarily supported by previous studies. $5,9,10,14$ We noticed that the nursing staff instructed the junior doctors comprising the no-EP group to use the standard PSA form, which possibly resulted in gradually better documentation of sedations in the absence of EPs. At the end of the study period, the form was used in over one tenth of the no-EP sedations.

\section{Conclusion}

PSA performed by EPs results in superior documentation of screening, used medication, and vital signs. There were similar success rates of the procedure in both groups. High quality documentation is particularly relevant in 
a vulnerable population, such as patients with dislocated hips. Our study suggests that the presence of EPs and the use of a standard sedation form improve the quality of documentation of sedation in the ED.

\section{Ethics}

This study was reviewed, approved and granted exempt status by the regional Ethical Review Board (METC, Southwest Holland, nr. 17-026). The requirement for participation consent was waived, by the Review Board, as all information was collected as part of routine clinical care and anonymized for the purpose of this observational study. This study conformed to the principles of the Declaration of Helsinki as it only consists of observational data research, without the involvement of any interventions to the patients.

\section{Acknowledgments}

The authors thank Crispijn L. van den Brand, Mariske Toonen, and Thomas Vissers for their critical appraisal of early versions of this study, and also Susy and Tod Hubert van Beusekom for their linguistic advice.

\section{Disclosure}

The authors report no conflict of interest in this work.

\section{References}

1. Burton JH, Miner JR, Shipley ER, et al. Propofol for emergency department procedural sedation and analgesia: a tale of three centers. Acad Emerg Med. 2006;13(1):24-30. doi:10.1197/j.aem.2005.08.011

2. Dutch institute for healthcare improvement (CBO). Guideline: sedation and/or analgesia (PSA) in locations outside the operating theater. Part 1: In adults. [In Dutch]. CBO. 2012;1-343. Available from: https://www. nvsha.nl/files/31/cbo_richtlijn_psa_volwassenen.pdf. AccessedMarch 27, 2021.

3. Ferguson I, Bell A, Treston G, et al. Propofol or ketofol for procedural sedation and analgesia in emergency medicine-The POKER Study: a Randomized Double-Blind Clinical Trial. Ann Emerg Med. 2016;68(5):574-582. doi:10.1016/j.annemergmed.2016.05.024

4. Frazee BW, Park RS, Lowery D, et al. Propofol for deep procedural sedation in the ED. Am J Emerg Med. 2005;23(2):190-195. doi:10.1016/j.ajem.2004.05.008

5. Kuypers MI, Plotz FB, Mencl F. Implementation strategies for procedural sedation and analgesia in the emergency department. Int J Emerg Med. 2017;10(1):6. doi:10.1186/s12245-017-0130-2

6. Kuypers MI, Smits GJP, Valkenet SC, et al. Procedural sedation and analgesia practices by emergency physicians in the Netherlands: a nationwide survey. Int $J$ Emerg Med. 2017;10(1):33. doi:10.1186/ s12245-017-0159-2
7. Kuypers MI, Smits GJP, Baerends EP, et al. Paediatric procedural sedation and analgesia by emergency physicians in a country with a recent establishment of emergency medicine. Eur J Emerg Med. 2019;26(3):168-173. doi:10.1097/MEJ.0000000000000524

8. Elkhodair SM, Baker EC, Glasebrook WR, et al. Emergency department procedural sedation: the London experience. Eur J Emerg Med. 2015;22(6):407-412. doi:10.1097/MEJ.0000000000000197

9. Celenza A, Winton J, Jackson T. Improving documentation of procedural sedation in an adult emergency department. Eur J Emerg Med. 2011;18(1):13-18. doi:10.1097/MEJ.0b013e328338db7e

10. Winton J, Celenza A, Jackson T. Improving documentation of endotracheal intubation in an adult emergency department. Emerg Med Australas. 2008;20(6):488-493. doi:10.1111/j.1742-6723.2008.011 34.x

11. Gaakeer MI, Veugelers R, Patka P, et al. Minimum operational standards for $24 / 7$ available emergency departments in the Netherlands: a first step taken by emergency physicians using an e-Delphi approach. Eur J Emerg Med. 2019;26(2):86-93. doi:10. 1097/MEJ.0000000000000494

12. Smits GJ, Kuypers MI, Mignot LA, et al. Procedural sedation in the emergency department by Dutch emergency physicians: a prospective multicentre observational study of 1711 adults. Emerg Med J. 2017;34(4):237-242. doi:10.1136/emermed-2016-205767

13. Thijssen WA, Giesen PH, Wensing M. Emergency departments in The Netherlands. Emerg Med J. 2012;29(1):6-9. doi:10.1136/ emermed-2011-200090

14. Kuypers MI, Mencl F, Verhagen MF, et al. Safety and efficacy of procedural sedation with propofol in a country with a young emergency medicine training program. Eur J Emerg Med. 2011;18 (3):162-167. doi:10.1097/MEJ.0b013e32834230fb

15. Reed MJ, Dunn MJ, McKeown DW. Can an airway assessment score predict difficulty at intubation in the emergency department? Emerg Med J. 2005;22(2):99-102. doi:10.1136/emj.2003.008771

16. Kuypers MI, Klijn A, Mullaart-Jansen NE, et al. Availability and quality of procedural sedation and analgesia in emergency departments without emergency physicians: a national survey in the Netherlands. World J Emerg Med. 2020;11(2):69-73. doi:10.5847/ wjem.j.1920-8642.2020.02.001

17. American Society of Anesthesiologists Task Force on Sedation and Analgesia by Non-Anesthesiologists. Practice guidelines for sedation and analgesia by non-anesthesiologists. Anesthesiology. 2002;96 (4):1004-1017. doi:10.1097/00000542-200204000-00031

18. Green SM, Krauss B. Propofol in emergency medicine: pushing the sedation frontier. Ann Emerg Med. 2003;42(6):792-797. doi:10.1016/ S0196-0644(03)00746-7

19. Heuss LT, Schnieper P, Drewe J, et al. Safety of propofol for conscious sedation during endoscopic procedures in high-risk patients-a prospective, controlled study. Am J Gastroenterol. 2003;98(8):1751-1757. doi:10.1111/j.1572-0241.2003.07596.x

20. Miner JR, Martel ML, Meyer M, et al. Procedural sedation of critically ill patients in the emergency department. Acad Emerg Med. 2005;12(2):124-128. doi:10.1197/j.aem.2004.08.054

21. Green SM, Roback MG, Miner JR, et al. Fasting and emergency department procedural sedation and analgesia: a consensus-based clinical practice advisory. Ann Emerg Med. 2007;49(4):454-461. doi:10.1016/j.annemergmed.2006.08.017 


\section{Publish your work in this journal}

Drug, Healthcare and Patient Safety is an international, peer-reviewed open-access journal exploring patient safety issues in the healthcare continuum from diagnostic and screening interventions through to treatment, drug therapy and surgery. The journal is characterized by the rapid reporting of reviews, original research, clinical, epidemiological and post-marketing surveillance studies, risk management, health literacy and educational programs across all areas of healthcare delivery. The manuscript management system is completely online and includes a very quick and fair peer-review system. Visit http://www.dovepress.com/testimonials.php to read real quotes from published authors. 